\title{
Does modifying the particle size distribution of a granular material (i.e., ma- terial scalping) alters its shear strength?
}

\author{
Emilien Azéma ${ }^{1, \star}$, Sandra Linero ${ }^{2, \star \star}$, Nicolas Estrada ${ }^{3, \star \star \star}$, and Arcesio Lizcano ${ }^{4, \star \star \star \star}$ \\ ${ }^{1}$ LMGC, Université de Montpellier, Montpellier, France \\ ${ }^{2}$ SRK Consulting Perth, Australia /University of Newcastle, Newcastle, Australia \\ ${ }^{3}$ Departamento de Ingeniería Civil y Ambiental, Universidad de Los Andes, Bogota, Colombia \\ ${ }^{4}$ SRK Consulting Vancouver, Canada
}

\begin{abstract}
By means of two dimensional contact dynamics simulations, we analyzed the effect of the particle size distribution (PSD) on the shear strength of granular materials composed of un-breakable disks. We modelled PSDs with a normalized beta function, which allows for building S-shaped gradation curves, such as those that typically occur in soils. We systematically controlled and varied the size span and the shape of the PSD, and found that the shear strength is independent both characteristics. This implies that PSD modification procedures such as material scalping (i.e., removing the smallest and/or largest particles in the sample) should not affect significantly the shear strength of the material composed of unbreakable discs. In order to explore the origins of the invariance of the shear strength with PSD, we analyzed the connectivity, force transmission, and friction mobilization in terms of anisotropies, finding that the constant shear strength is due to a subtle compensation of anisotropies.
\end{abstract}

\section{Introduction}

The study of the mechanical behavior of mine wastes containing particles of metric order is a challenging task because commercial testing devices can only accommodate samples composed of particles a few centimeters in dimension. The construction of larger devices for laboratory testing is not economical. To overcome the equipment size limitations, testing of coarse material is conducted on samples prepared by convenient modification of the particle size distribution (PSD). Parallel scaling (i.e., reducing the particles' size while keeping the PSD shape constant) or material scalping (i.e., removing the smallest and/or largest particles) are some of the technics used to prepare samples for laboratory testing [1]. The tested sample may thus differ from the prototype in both the size of the particles and the form of the PSD.

Sample preparation procedures such as parallel scaling or material scalping are widely used in engineering practice. However, when and how these procedures affect the material's mechanical response (e.g., the shear strength) remains an open question. Important elements to be considered for tackling this question are: (i) the relation between particle size and fragmentation probability; (ii) the link between PSD, packing fraction, and connectivity; and (iii) the evolution of particle's shape and interaction's forces with particle size.

\footnotetext{
^ e-mail: emilien.azema@umontpellier.fr

$\star \star$ e-mail: slinero@srk.com.au

$\star \star \star$ e-mail: n.estrada22@uniandes.edu.co

$\star \star \star \star$ e-mail: alizcano@srk.com
}

A possible strategy for studying this problem theoretically is to analyze simplified systems (e.g., twodimensional systems, comprised of circular and unbreakable grains, with simple interaction laws such as Coulomb friction). This allows for singling out the effects of the PSD while leaving out the "noise" of other important factors. Discrete element methods are well suited for this type of simulations. Recently, this strategy has been used successfully to analyze the effect of the PSD on packing fraction and shear strength (see Refs. [2-6]), focusing on the comparison between bi-disperse and continuous PSDs, as well as on uniform distributions by volume fraction. Works exploring the effect of the PSD shape by means of discrete element simulations are rare (see Refs. $[7,8])$.

The main objective of this work was to analyze the effect of the particle size distribution (PSD) on the shear strength of granular materials composed of unbreakable disks. We build different PSDs using a normalized beta function, which allows for building S-shaped gradation curves, such as those that typically occur in soils. We varied two parameters independently over a broad parametric space, controlling both the size span and the shape of the PSD. We found that the shear strength is invariant with both the size span and the shape of the PSD. From a micromechanical viewpoint, we show that the shear strength remains constant for different PSDs thanks to a subtle compensation of anisotropies, specifically, contact and branch length anisotropies. The results support several 
hypotheses proposed recently and extend their validity to a larger family of PSDs.

\section{Numerical procedures}

The size span of the PSDs is defined by:

$$
s=\left(d_{\max }-d_{\min }\right) /\left(d_{\max }+d_{\min }\right),
$$

where $d_{\max }$ and $d_{\min }$ are the minimum and maximum particle diameters, respectively; $s=0$ corresponds to a monodisperse packing whereas $s=1$ corresponds to an infinitely large polydispersity.

A $\beta$-function was use to model the shape of the PSDs. It is defined by:

$$
\beta(x)=\frac{1}{B(a, b)} \int_{0}^{x} t^{a-1}(1-t)^{b-1} d t,
$$

where, $a>0$ and $b>0$ are the parameters of the distribution and $B(a, b)=\Gamma(a) \Gamma / \Gamma(a+b)$, where $\Gamma$ is the Gamma function. The PSD can then be described using the cumulate distribution function $h(d)$ (also called grading curve in industrial contexts), given by

$$
h(d)=\beta\left[x=d_{r}(d) ; a, b\right],
$$

where $d_{r}(d)=\left(d-d_{\text {min }}\right) /\left(d_{\text {max }}-d_{\text {min }}\right)$ is the the reduced diameter. As shown by Voivret et al. in Ref. [9], this model allows for controlling both the size span and the shape of the PSD, including reference distributions such as monodisperse, power law, and S-shaped PSD curves. In our simulations, $d_{\text {max }}$ was $0.15 \mathrm{~m}$ and the size span was varied in the range $s \in[0.2,0.4,0.6,0.8,0.9]$.

In order to generate a set of particle diameters following a given distribution, the range $d_{\max }-d_{\min }$ was divided into $N_{c}$ classes. Within each class, a uniform distribution by volume (area) of grains was considered. By construction, the number of particles increased with $b$, thus $N_{c}$ and the total number of particles $N_{p}$ were chosen in order to: (i) obtain a good fit with the theoretical curve and (ii) obtain a "reasonable" sample size, in terms of the number of particles. In the simulations presented here, the parameter $a$ was fixed to 1 and the parameter $b$ varied from 1 (a uniform distribution by volume fraction) to 5 (S-shaped gradation with a pronounced curvature). The total number of particles increased from 10,000 for $b=1$ to $\sim 70,000$ for $b=5$. Figure 1 shows the theoretical PSDs, i.e., Eq.3, as well as the PSD of the simulated samples (points), for several combinations of $s$ and $b$.

The simulations used the contact dynamics (CD) method [10], which assumes perfectly rigid particles interacting through mutual exclusion and Coulomb friction. We used the code LMGC90 which is a multipurpose software developed in the University of Montpellier, capable of modeling a collection of deformable or undeformable particles of various shapes by different algorithms. In each simulation, the particles were first compacted by isotropic compression inside a box. The gravity was set to 0 . The friction coefficients between particles was set to $\mu=0.4$. The friction coefficient between the particles and the walls

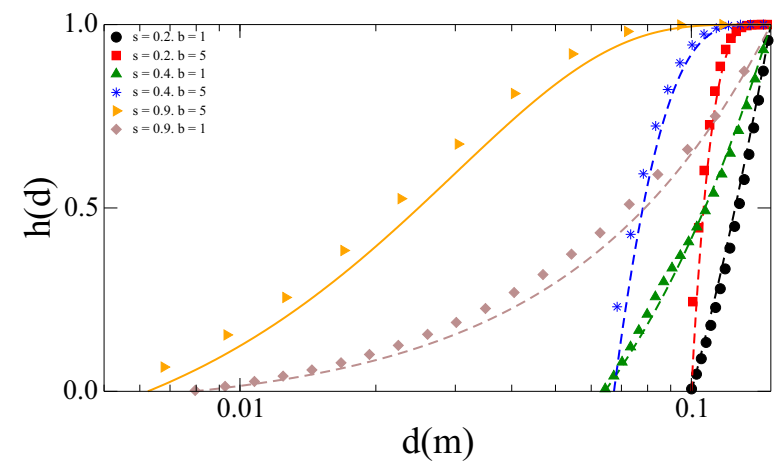

Figure 1. Theoretical and simulated particle size distributions for several combinations of parameters $s$ and $b$.

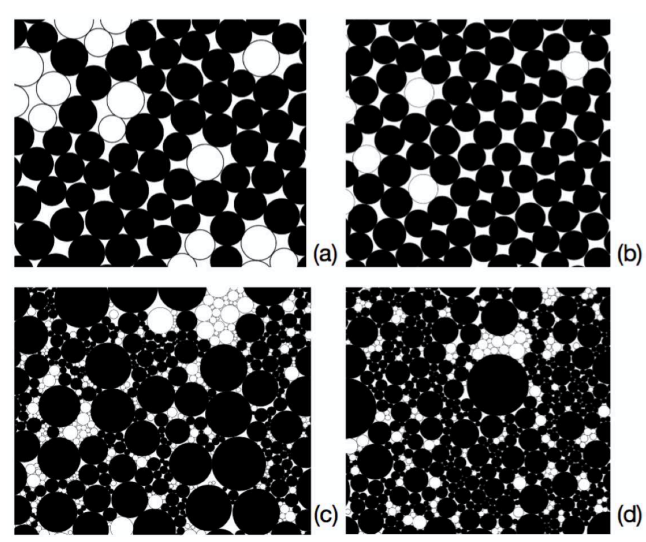

Figure 2. Zoom for $(s=0.2, b=1)(\mathrm{a}),(s=0.2, b=5)(\mathrm{b})$, $(s=0.9, b=1)(\mathrm{c})$ and $(s=0.9, b=5)(\mathrm{d})$ at the end of the isotropic compression. Floating particles (i.e., particles with one or no contacts) are shown in white.

was set to $\mu_{w}=0$, in order to obtain homogeneous and isotropic particles packing. Figure 2 shows zooms of the packings obtained for four different values of $s$ and $b$ at the end of isotropic compaction. Small crystalized regions can be observed for small values of $s$, which happens because most of the particles have the same diameter. In contrast, disorder increases with $s$.

The samples were then sheared in a biaxial configuration. A downward velocity $v_{y}$ was imposed on the upper wall while keeping a constant confining stress on the lateral walls. The strain rate $v_{y} / H$ was low, so that the test can be considered as quasi-static.

\section{Macroscopic shear strength}

The stress tensor $\sigma$ can be evaluated from the simulation data as an average over all the contacts of the dyadic product of the contact force $\mathbf{f}^{\mathbf{c}}$ and the branch vector $\ell^{\mathbf{c}}$ : $\sigma_{\alpha \beta}=n_{c}\left\langle f_{\alpha}^{c} \ell_{\beta}^{c}\right\rangle_{c}$ [10], where $n_{c}$ is the number density of contacts $c$ and the average $\langle\ldots\rangle_{c}$ runs over all contacts in a control volume. In $2 \mathrm{D}$, we define the stress deviator as $q=\left(\sigma_{1}-\sigma_{2}\right) / 2$ and the mean stress as $p=\left(\sigma_{1}+\sigma_{2}\right) / 2$, where $\sigma_{1,2}$ are the principal stresses. The stress state is 


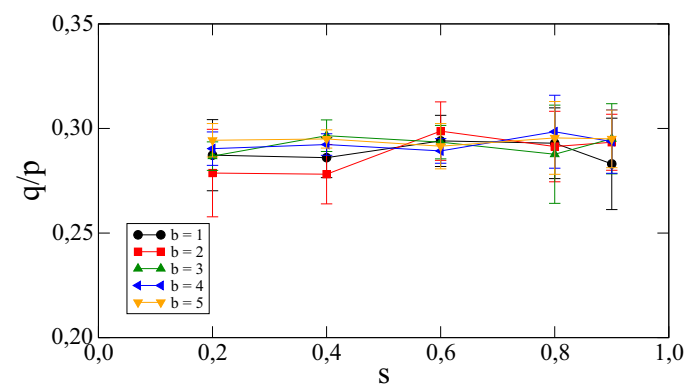

Figure 3. Shear strength $(q / p)^{*}$ in the steady state as function of $s$, for all values of $b$.

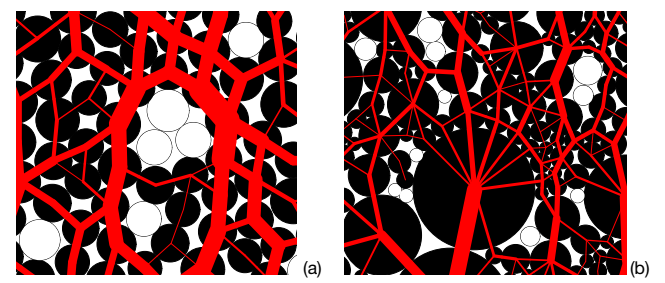

Figure 4. Maps of normal forces in a portion of the sample for $(s=0.2, b=5)(\mathrm{a})$ and $(s=0.9, b=5)(\mathrm{b})$ after a large shear strain. Line thickness is proportional to the normal force.

described by the mean stress $p$ and the normalized shear stress $q / p$ [11]. During shear, $q / p$ increased initially to a peak value and then decreased to a nearly constant value $(q / p)^{*}$, which we used to characterize the material's shear strength in the steady state.

Figure 3 shows the evolution of $(q / p)^{*}$ as a function of $s$, for all values of $b$. It can be seen that, regardless of $b$, the shear strength is almost independent of $s$ and close to 0.28 . This has been previously observed for samples composed of circular [12] and polygonal [6] particles with a uniform distribution by volume fraction. Our results extend this counterintuitive observation to the family of Sshaped PSDs described by Eq. 3.

\section{Anisotropies of the contact and force network}

The shear strength of dry granular materials is generally attributed to the buildup of an anisotropic structure during shear due to friction between the particles and as a result of steric effects depending on particle shape [13].This is well illustrated in Fig. 4, which shows two maps of normal forces in a portion of the sample for $(s, b)=(0.2,5)$ and $(s, b)=(0.9,5)$ after a large shear strain. The contact forces are represented by segments joining the particle centers, whose thickness is proportional to the force magnitude. Visual inspection reveals that the connectivity of the contact network varies drastically with $s$ in terms of both the mean number of contacts per particle (i.e., coordination number) and the contacts' orientation and length.

A way to capture this microstructure is to consider the probability distribution $P(\boldsymbol{n})$ of the contact normals $\boldsymbol{n}$, which is generically nonuniform. In two dimensions, the unit vector $\boldsymbol{n}$ is described by a single angle $\theta$, the orientation of the contact normal. The probability density function $P(\theta)$ of contact normals provides detailed statistical information about the fabric. It is $\pi$-periodic in the absence of an intrinsic polarity for $\boldsymbol{n}$. At the lowest order, we have $[14,15]$

$$
P(\theta)=\left\{1+a_{c} \cos 2\left(\theta-\theta_{\sigma}\right)\right\} / 2 \pi,
$$

where $\theta_{\sigma}$ is the major principal stress direction $\left(\theta_{\sigma}=\pi / 2\right)$, and $a_{c}$ is the contact anisotropy.

This description of the granular microstructure can be enriched by considering also the angular average of normal branch length and forces:

$$
\left\{\begin{array}{l}
\left\langle\ell_{n}\right\rangle(\theta)=\langle\ell\rangle\left\{1+a_{\ell n} \cos 2\left(\theta-\theta_{\sigma}\right)\right\} \\
\left\langle f_{n}\right\rangle(\theta)=\left\langle f_{n}\right\rangle\left\{1+a_{f n} \cos 2\left(\theta-\theta_{\sigma}\right)\right\} \\
\left\langle f_{t}\right\rangle(\theta)=\left\langle f_{n}\right\rangle a_{f t} \sin 2\left(\theta-\theta_{\sigma}\right)
\end{array}\right.
$$

where $\left\langle f_{n}\right\rangle$ and $\langle\ell\rangle$ are the mean force and the mean branch length, and $a_{l n}, a_{f n}$, and $a_{f t}$ the branch length, normal force and tangential forces anisotropies.

The anisotropies $a_{c}, a_{\ell n}, a_{f n}$, and $a_{f t}$ are interesting descriptors of the granular microstructure and force transmission properties, because they underlie the different microscopic origins of shear strength. The general expression of the stress tensor leads to the following simple relation $[14,15]$ :

$$
\frac{q}{p} \simeq \frac{1}{2}\left(a_{c}+a_{l n}+a_{f n}+a_{f t}\right)
$$

This expression is very useful, since it allows for describing the shear strength as the addition of several microstructural anisotropies. It is worth noting that the shear strength can be understood as the material's ability to bear anisotropic stress.

Figure 5(a) shows the evolution of $a_{c}$ and $a_{\ell n}$ as functions of $s$, for all values of $b$. For small values of $b$ (i.e., $b=1$ and 2), it can be seen that $a_{c}$ decreases with $s$, indicating that the anisotropy of the contact network is decreasing. This happens because, as $s$ increases, the coordination number also increases, and the particles are surrounded by an increasing number of contacts. On the other side, it can be seen that $a_{\ell n}$ increases with $s$, indicating that the branch lengths of the contacts that are oriented along the principal stress direction tend to be longer. These results show that the shear strength remains constant due to a compensation of anisotropies: the contact network becomes less anisotropic but the force chains along the principal stress direction tend to be captured by the larger particles. Lastly, for large values of $b$ (i.e., $b=4$ and 5), both $a_{c}$ and $a_{\ell n}$ remain approximately constant with $s$ and $b$.

Figure 5(b) shows the evolution of $a_{f n}$ and $a_{f t}$ as functions of $s$ for all values of $b$. In contrast to contact and branch length anisotropies, it can be seen that $a_{f n}$ and $a_{f t}$ are approximately constant with $s$ and $b$. This means that the anisotropy of force chains as well as the degree of friction mobilization are not affected by changing the PSD. 


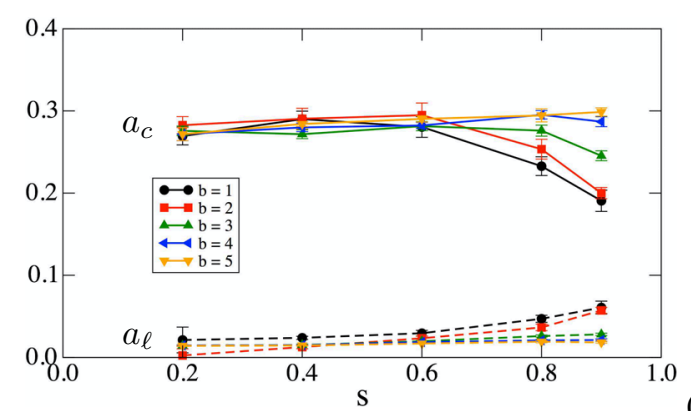

(a)

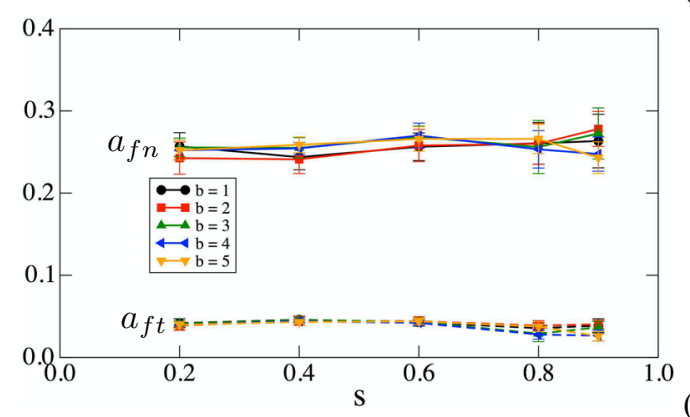

(b)

Figure 5. (a) Contact and branch length anisotropies, $a_{c}$ and $a_{\ell n}$ respectively, and (b) Normal and tangential force anisotropies, $a_{f n}$ and $a_{f t}$ respectively, all averaged in the steady state, as functions of $s$, for all values of $b$.

\section{Conclusions}

We investigated the joint effect of the size span and shape of the particle size distribution (PSD) on the shear strength of packings composed of unbreakable disks, by means of contact dynamics simulations. For this, we independently varied the size span and shape of the distribution across a broad parametric space, and investigated the micromechanical origin of the shear strength for each simulated PSD.

On the first hand, we showed that the shear strength is independent of both the size span and the shape of the PSD. This has already been observed, also by means of discrete element simulations, for samples with uniform distributions by volume fraction $[6,12]$. In these cases, only the PSD size span was varied in samples with uniform distributions by volume fraction. Our results extend the validity of this result by showing that the shear strength is invariant, not only with the size span, but also with the shape of the PSD, at least for those shapes that can be described by Eq. 3. These findings are in agreement with those reported recently in [8] for particle distributions described by a power law.

On the second hand, we investigated the origins of the shear strength by analyzing the contact, branch length, and force anisotropies. It was shown that the shear strength remains constant due to a compensation between the contact and branch length anisotropies, while force anisotropy and friction mobilization remain constant. As shown previously $[6,12]$, these results confirm that, as the size span of the PSD increases, the system is more and more connected and larger forces tend to be captured by larger particles.

The results suggest that the differences observed on the laboratory, in the mechanical response of prototype soil samples and its scaled representations, can be attributed to factors other than the PSD changes introduced with the scaling procedure. It should be noted that this investigation considers that particle characteristics (e.g., shape, strength, and interaction laws) do not depend on particle size. In real materials, several important characteristics, such as grain fragmentation probability, grain shape, and interaction forces, depend notably on particle size. As a consequence, modifying the PSD of a real soil change also the nature of the particles that compose the sample, which can provoke appreciable changes in the mechanical response of the granular system.

\section{References}

[1] J. Duncan, S. Wright, in Soil Strength and Slope Stability, edited by J. Wiley \& Sons (2005), ISBN 9780471691631

[2] M. Wackenhut, H. Herrmann, Physical Review E 68, 041303 (2003)

[3] C. Voivret, Ph.D. thesis, Université de Montpellier (2009)

[4] B. Yohannes, K.M. Hill, Physical Review E 82, 1 (2010)

[5] D.H. Nguyen, E. Azéma, F. Radjai, P. Sornay, Physical Review E 90, 012202 (2014)

[6] D.H. Nguyen, E. Azéma, P. Sornay, F. Radjai, Physical Review E 91, 1 (2015)

[7] P. Stroeven, M. Stroeven, Cement and Concrete Research 29, 1201 (1999)

[8] N. Estrada, Physical Review E Accepted (2016)

[9] C. Voivret, F. Radjaï, J.Y. Delenne, M. El Youssoufi, Physical Review E 76, 1 (2007)

[10] J.J. Moreau, European Journal of Mechanics A/Solids 13, 93 (1994)

[11] J. Mitchell, K. Soga, Fundamentals of Soil behavior (John Wiley, 2005), ISBN 978-0-471-46302-3

[12] C. Voivret, F. Radjaï, J.Y. Delenne, M. El Youssoufi, Physical Review Letters 102, 178001 (2009)

[13] F. Radjai, J.Y. Delenne, E. Azéma, S. Roux, Granular Matter 14, 259 (2012)

[14] L. Rothenburg, R.J. Bathurst, Geotechniques 39, 601 (1989)

[15] E. Azéma, N. Estrada, F. Radjaï, Physical Review E 86, 041301 (2012) 